\title{
Pain-motor integration in the primary motor cortex in Parkinson's disease
}

\author{
A. Suppa a, b, C. Leone a , F. Di Stasio b, L. Marsili a , A. Di Santo ${ }^{\text {a, c }, ~ A . ~ B i a s i o t t a ~}{ }^{\text {a }, ~ S . ~ L a ~ C e s a ~}{ }^{\text {a }}$, \\ A. Truini ${ }^{\text {a }}$, G. Cruccu ${ }^{\text {a }}$, A. Berardelli ${ }^{\text {a, b, * }}$ \\ a Department of Neurology and Psychiatry, Sapienza University of Rome, Italy \\ ${ }^{\mathrm{b}}$ IRCCS Neuromed Institute, Pozzilli IS, Italy \\ ${ }^{\mathrm{c}}$ Neurology Unit, Campus Bio-Medico University, Rome, Italy
}

\section{A R T I C L E I N F O}

\section{Article history:}

Received 27 October 2016

Received in revised form

7 February 2017

Accepted 27 April 2017

Available online $\mathrm{xxx}$

\section{Keywords:}

Laser-evoked potentials

Pain-motor integration

Paired associative stimulation

Parkinson's disease

Primary motor cortex

\begin{abstract}
A B S T R A C $T$
Background: In Parkinson's disease (PD), the influence of chronic pain on motor features has never been investigated. We have recently designed a technique that combines nociceptive system activation by laser stimuli and primary motor cortex (M1) activation through transcranial magnetic stimulation (TMS), in a laser-paired associative stimulation design (Laser-PAS). In controls, Laser-PAS induces long-term changes in motor evoked potentials reflecting M1 long-term potentiation-like plasticity, arising from pain-motor integration.

Objective: We here examined the possible influence of chronic pain on motor responses to Laser-PAS in patients with PD, with and without chronic pain.

Methods: We compared motor responses to Laser-PAS in healthy subjects and in patients with PD, with and without chronic pain.

Results: Unlike controls, we found reduced responses to Laser-PAS in patients with PD, with and without pain. Patients off and on dopaminergic therapy had similar responses to Laser-PAS. When comparing responses to Laser-PAS in patients with and without pain, the two patients' subgroups had similar abnormalities. When we compared patients with pain in the body region investigated with Laser-PAS, with those with pain in other body regions, we found prominent changes in patients with homotopic pain. Finally, when comparing Laser-PAS with the original PAS protocol, which combines electric peripheral nerve stimuli and TMS, in patients without pain and those with homotopic pain, we found similar responses to both techniques in patients without pain, whereas Laser-PAS induced greater abnormalities than PAS in patients with pain.

Conclusions: In PD, chronic pain degrades response to Laser-PAS through abnormal pain-motor integration.
\end{abstract}

() 2017 Elsevier Inc. All rights reserved.
Abbreviations: ACC, anterior cingulate cortex; ADM, abductor digiti minimi; ANOVA, analysis of variance; La DN4, douleur neuropatique en 4 questions; HAM-D, Hamilton Depression Rating Scale; Laser-PAS, laser-paired associative stimulation; LEDDs, L-Dopa equivalent daily doses; LEP, laser evoked potential; LTP, long-term potentiation; LTD, long-term depression; M1, primary motor cortex; MEP, motor evoked potential; MMSE, Mini Mental State Evaluation; NRS, numerical rating scale; PAS, paired associative stimulation; PD, Parkinson's disease; RMT, resting motor threshold; S2, secondary somatosensory area; STDP, spike timing-dependent plasticity; TMS, transcranial magnetic stimulation; MDS-UPDRS, Movement Disorders Society-Unified Parkinson's Disease Rating Scale.

* Corresponding author. Department of Neurology and Psychiatry, Neuromed Institute, Sapienza University of Rome, Viale dell'Università, 30, 00185 Rome, Italy.

E-mail address: alfredo.berardelli@uniroma1.it (A. Berardelli).
Chronic pain is a non-motor symptom observed in $40-85 \%$ of patients with Parkinson's disease (PD) [1-4]. In PD, chronic pain is currently classified in nociceptive (i.e. muscoloskeletal, visceral and cutaneus pain) and neurophatic pain (i.e. radicular and central pain) $[1,5,6]$. Similarly to other non-motor symptoms in PD, pain is currently considered to reflect extranigral pathology $[7,8]$, but the pathophysiology of chronic pain in PD remains largely unknown $[1,2,5,6,9]$.

Laser stimulation is widely considered a reliable tool for investigating pain pathways in humans [10]. Laser stimulation selectively activates $A \delta$ and C nociceptors and evokes [11] scalp potentials (LEPs) comprising an early lateralized component (N1), generated by secondary sensory area (S2) area and a late negative- 
positive complex (N2-P2) generated by anterior cingulate cortex (ACC) [11-13]. Previous studies in patients with PD demonstrated LEP abnormalities in patients with nociceptive and neuropathic pain $[14,15]$, suggesting abnormal cortical processing of nociceptive inputs as a key pathophysiological mechanism underlying chronic pain in PD $[2,4,9]$.

Recently we have designed a new technique, namely Laserpaired associative stimulation (Laser-PAS 50 ) [16]. This protocol combines laser pulses delivered to the hand with transcranial magnetic stimulation (TMS) of M1 in a PAS design. In healthy subjects, Laser-PAS 50 elicits long-term increase of motor evoked potential (MEP) amplitude through mechanisms of long-term potentiation (LTP)-like plasticity in M1 possibly related to changes in $N$-Methyl-D-aspartate transmission [16].

No studies have previously investigated M1 plasticity as assessed with the Laser-PAS ${ }_{50}$ technique in PD with and without chronic pain. A better understanding of pain-related M1 plasticity might clarify the pathophysiological basis of chronic pain in PD and open new perspectives in the treatment of this non-motor symptom.

In this study, we applied Laser-PAS 50 in a cohort of patients with PD. Then to clarify whether in PD chronic pain influences the responses to Laser-PAS 50 , we compared Laser-PAS 50 in patients with and without pain. To understand whether pain has a segmental or generalized effect, we compared responses to Laser-PAS 50 in patients with pain in the right upper limb, the same body region examined by Laser-PAS 50 , and patients with pain in other body regions. Finally, to verify whether abnormal responses to Laser$\mathrm{PAS}_{50}$ in patients with chronic pain depends on intrinsic M1 plasticity abnormalities regardless of the specific PAS protocol used, we compared responses to Laser-PAS 50 and to the original PAS protocol, which combines electric peripheral nerve stimuli and transcranial magnetic stimulation (TMS) at interstimulus interval of $25 \mathrm{~ms}\left(\mathrm{PAS}_{25}\right)$ [17], in the same cohort of patients, with and without pain.

\section{Material and methods}

\section{Subjects}

Twenty patients with PD (14 men and 6 women, mean age \pm SD: $62.5 \pm 9.5$ ) and 20 age-matched healthy subjects (10 men and 10 women, mean age \pm SD: $59.7 \pm 15.8$ ) participated in the study. All participants were right-handed. The diagnosis of idiopathic PD was made according to the Queen Square Brain Bank criteria and the EFNS/MDS-ES recommendations [18,19]. Patients were recruited from the Movement Disorder Clinic at the Department of Neurology and Psychiatry, Sapienza University of Rome. Patients had a predominantly akinetic-rigid syndrome without dementia. Patients were studied while they were under their usual dopaminergic treatment (on) and after drugs had been withdrawn for at least $12 \mathrm{~h}$ (off). None of the patients received other neuropsychiatric medications. Patients were clinically evaluated before starting each experimental session. Motor signs were scored using the motor section of the Movement Disorders Society-Unified Parkinson's Disease Rating Scale (MDS-UPDRS) $[20,21]$ and the Hoehn \& Yahr (H\&Y) scale. Cognitive function was evaluated using the Mini Mental State Evaluation (MMSE) [22] and the Frontal Assessment Battery (FAB) [23]. Depression was assessed with the Hamilton Depression Rating Scale (HAM-D) [24]. According to the criteria suggested by Wasner et al. [5], we first evaluated the presence of chronic pain. None of the healthy controls reported any type of pain. Patients were asked to rate the intensity of pain on an 11point numerical rating scale (NRS) from 0 (no pain) to 10 (strongest imaginable pain) [10] before the first experimental session and relative to the previous 6 months. Those rating pain $\geq 4$ and reporting it from at least 6 months were considered "chronic-pain" patients. Patients with chronic pain also underwent the screening tool for the detection of neuropathic pain (La douleur neuropatique en 4 questions - DN4) [25]. No patients were being administered with any treatment for pain at the time of the study. Demographic and clinical features of parkinsonian patients, with and without pain are summarized in Table 1. Subjects gave their written informed consent. The study was approved by the institutional review Board of Sapienza, University of Rome, Italy and conformed to the Declaration of Helsinki.

\section{Laser stimulation technique and LEP recordings}

Laser stimuli were delivered with a neodymium:yttriumaluminium-perovskite laser stimulator (Nd:YAP, wave length $1.34 \mu \mathrm{m}$, pulse duration 2-20 ms, maximum energy $7 \mathrm{~J}$ El.En Florence, Italy) under fiber-optic guidance. The laser perceptive threshold was determined by increasing and decreasing the stimulus energy in steps of $0.25 \mathrm{~J}$ in series of three stimuli, and defined as the lowest intensity at which the subjects perceived at least the $50 \%$ of the stimuli [26]. To evoke clear and stable LEPs, laser stimuli were set to induce a clear painful pinprick sensation (intensity 119.4-150 $\mathrm{mJ} / \mathrm{mm} 2$; duration $5 \mathrm{~ms}$; spot diameter $5 \mathrm{~mm}$ ) and directed to the ulnar region of the right hand dorsum. The target of the laser beam was shifted by at least $1 \mathrm{~cm}$ in a random direction, to allow for passive skin cooling and avoid nociceptor fatigue or sensitization and the interstimulus interval was varied pseudorandomly (10-15 s) [26]. Subjects, wearing protective goggles, rested comfortably on a medical examination table. LEPs were recorded through surface electrodes from the scalp: T3 referenced to $\mathrm{Fz}$ for recording the early lateralized $\mathrm{N} 1$ component, and $\mathrm{CZ}$ referenced to the nose, for recording the late vertex N2-P2 complex. Electro-oculographic recordings monitored possible eye movements or blinks. We averaged 15 artefact-free trials off line using dedicated equipment (Synergy 10.1; EL.EN, Florence, Italy). The recording bandpass was between 0.3 and $30 \mathrm{~Hz}$ and the sampling frequency $50 \mathrm{kHz}$. We measured the peak latency and amplitude of the lateralized N1 and the vertex N2-P2 complex. These methods adhered to the recommendations given by the International Federation of Clinical Neurophysiology [11]. LEP recordings were taken at least $30 \mathrm{~min}$ before TMS, while the patients were on therapy ( $1 \mathrm{~h}$ after taking their usually antiparkinsonian therapy).

\section{TMS and MEP recordings}

TMS was delivered through a repetitive magnetic stimulator (Magstim Super Rapid-The Magstim Company Ltd, Whitland, United Kingdom) connected to a figure-of-eight coil (external wing $9 \mathrm{~cm}$ in diameter) placed tangentially to the scalp on the left hemisphere, with the handle pointing back and away from the midline at $45^{\circ}$ inducing postero-anterior and antero-posterior biphasic currents in the brain. The coil was placed over the optimum scalp position (hot spot) to elicit MEPs in the abductor digiti minimi (ADM) muscle of the right hand. To ensure that the stimulating coil remained in a constant position throughout the experiments, the hot spot was marked on the scalp with a soft-tipped pen. Motor threshold was determined at rest (RMT) as the lowest intensity able to evoke a MEP of more than $50 \mu \mathrm{V}$ in at least 5 of 10 consecutive trials in the ADM muscle. RMT was determined in steps of $1 \%$ maximum stimulator output intensity. Electromyographic activity was recorded through a pair of surface electrodes placed over the right ADM muscle, using a belly-tendon montage. Electromyographic raw signals were recorded, sampled at $5 \mathrm{kHz}$ with a CED $1401 \mathrm{~A} / \mathrm{D}$ laboratory interface (Cambridge Electronic Design, 
Table 1

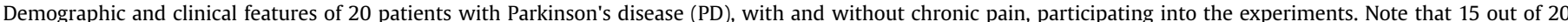

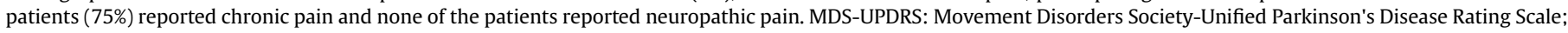

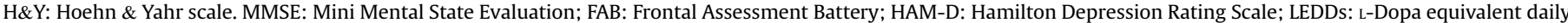

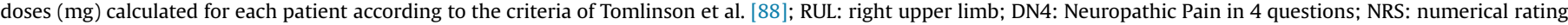
scale for pain; AV: average. SD: standard deviation. The 0 scores from the questionnaire for the detection of neuropathic pain (DN4) are not shown.

\begin{tabular}{|c|c|c|c|c|c|c|c|c|c|c|c|c|c|c|}
\hline \multirow[t]{2}{*}{ Patient } & \multirow{2}{*}{$\begin{array}{l}\text { Age } \\
\text { (years) }\end{array}$} & \multirow[t]{2}{*}{ Gender } & \multirow[t]{2}{*}{ Disease Duration } & \multicolumn{2}{|c|}{ UPDRS } & \multirow[t]{2}{*}{$\mathrm{H} \& \mathrm{Y}$} & \multirow[t]{2}{*}{ MMSE } & \multirow[t]{2}{*}{ FAB } & \multirow[t]{2}{*}{ HAM-D } & \multirow[t]{2}{*}{ LEDDs } & \multirow[t]{2}{*}{ ChronicPain } & \multirow[t]{2}{*}{ RUL Pain } & \multirow[t]{2}{*}{ DN4 } & \multirow[t]{2}{*}{ NRS } \\
\hline & & & & On & Off & & & & & & & & & \\
\hline 1 & 71 & $\mathrm{M}$ & 1 & 22 & 32 & 2 & 28 & 16 & 2 & 300 & - & - & - & - \\
\hline 2 & 74 & M & 14 & 9 & 30 & 2 & 30 & 17 & 2 & 850 & + & - & 1 & 4 \\
\hline 3 & 69 & M & 5 & 26 & 40 & 2 & 24 & 14 & 5 & 650 & - & - & - & - \\
\hline 4 & 80 & M & 7 & 11 & 20 & 1 & 30 & 16 & 0 & 550 & + & + & 0 & 6 \\
\hline 5 & 48 & $\mathrm{~F}$ & 7 & 20 & 30 & 2 & 30 & 18 & 3 & 405 & + & - & 0 & 7 \\
\hline 6 & 61 & $\mathrm{~F}$ & 8 & 21 & 32 & 2 & 28 & 17 & 3 & 460 & - & - & - & - \\
\hline 7 & 63 & $\mathrm{~F}$ & 7 & 18 & 33 & 2 & 30 & 18 & 2 & 400 & + & + & 0 & 8 \\
\hline 8 & 59 & $\mathrm{M}$ & 1 & 4 & 12 & 1 & 30 & 18 & 7 & 310 & + & + & 0 & 4 \\
\hline 9 & 69 & $\mathrm{M}$ & 5 & 12 & 20 & 2 & 30 & 18 & 0 & 310 & - & - & - & - \\
\hline 10 & 60 & $\mathrm{M}$ & 2 & 10 & 12 & 1 & 30 & 18 & 0 & 260 & - & - & - & - \\
\hline 11 & 69 & $\mathrm{M}$ & 10 & 17 & 40 & 2 & 28 & 17 & 2 & 942 & + & + & 0 & 5 \\
\hline 12 & 63 & $\mathrm{M}$ & 7 & 8 & 22 & 1 & 30 & 18 & 3 & 400 & + & + & 0 & 6 \\
\hline 13 & 62 & $\mathrm{M}$ & 3 & 14 & 25 & 2 & 30 & 18 & 7 & 310 & + & - & 0 & 4 \\
\hline 14 & 60 & $\mathrm{~F}$ & 5 & 12 & 20 & 2 & 30 & 18 & 7 & 405 & + & - & 0 & 5 \\
\hline 15 & 58 & $\mathrm{M}$ & 1 & 11 & 16 & 2 & 30 & 18 & 7 & 160 & + & - & 0 & 5 \\
\hline 16 & 40 & $\mathrm{~F}$ & 3 & 35 & 38 & 1 & 30 & 18 & 20 & 310 & + & + & 3 & 5 \\
\hline 17 & 75 & $\mathrm{M}$ & 2 & 20 & 23 & 1 & 29 & 8 & 13 & 400 & + & + & 3 & 7 \\
\hline 18 & 61 & $\mathrm{M}$ & 1 & 22 & 26 & 1 & 30 & 18 & 4 & 400 & + & + & 0 & 5 \\
\hline 19 & 53 & $\mathrm{M}$ & 1 & 14 & 15 & 1 & 30 & 17 & 7 & 340 & + & + & 0 & 4 \\
\hline 20 & 55 & $\mathrm{~F}$ & 2 & 16 & 20 & 1 & 30 & 18 & 0 & 300 & + & + & 0 & 5 \\
\hline Av & 62.5 & & 4.6 & 16.1 & 25.3 & 1.6 & 29.4 & 16.9 & 4.7 & 423.1 & & & 0.5 & 5.3 \\
\hline SD & 9.5 & & 3.6 & 7.2 & 8.8 & 0.5 & 1.5 & 2.3 & 4.9 & 193.0 & & & 1.1 & 1.2 \\
\hline
\end{tabular}

Cambridge, UK), amplified $(\times 1000)$, and filtered (bandwidth 5 $1 \mathrm{kHz}$ ) with the Digitimer D360 (Digitimer Ltd., Welwyn Garden City, Hertfordshire, UK) and stored on a laboratory computer for online visual display and further off-line analysis (Signal software, Cambridge Electronic Design, Cambridge, UK). The baseline electromyographic activity level before, during and after TMS was controlled by visual-feedback through an oscilloscope screen and auditory feedback through a loudspeaker. To exclude possible confounding effects from involuntary muscular contraction, trials with background electromyographic activity were rejected. MEP amplitude was measured peak-to-peak $(\mathrm{mV})$ and then averaged.

\section{Experimental design}

The study consisted of two separate experimental sessions (Experiments 1-2; Fig. 1). All controls and patients participated in Experiment 1, whereas a subgroup of 10 patients participated in Experiments 2. All experimental sessions took place at comparable daytime and at least 1 week elapsed between each session. Participants were tested fully relaxed and with their eyes open.

\section{Experiment 1: Effect of Laser-PAS 50}

In this experiment, designed to investigate the response to Laser-PAS 50 protocol in healthy controls and patients with PD, with and without pain, when off and on therapy, we used a "test-conditioning" TMS approach. The "test" stimulation consisted of 20 single TMS pulses able to elicit MEPs of $1 \mathrm{mV}$ from the right ADM at baseline (T0). We then recorded and measured a clear-cut peak LEP $\mathrm{N} 1$ component according to the international recommendations [11]. We applied "conditioning" Laser-PAS 50 consisting of 60 repetitive TMS pulses at $0.1 \mathrm{~Hz}$, each TMS pulse following a single laser stimulus delivered at an interstimulus interval of LEP N1 latency $+50 \mathrm{~ms}$ (total duration of intervention: $10 \mathrm{~min}$ ) [16]. Laser stimuli were delivered at the same intensity used during LEP recording. To investigate the time course of after-effects induced by
Laser-PAS 50 , we compared baseline MEPs (T0) with those recorded immediately after Laser-PAS ${ }_{50}$ ended (T1) and at 10 (T2), 20 (T3), 30 (T4), 40 (T5), 50 (T6) and 60 (T7) after it ended. All patients were studied at T1-T7. Patients were randomly assigned to participate in two separate sessions, when off and on therapy.

\section{Experiment 2: Effect of Laser-PAS 50 and $\mathrm{PAS}_{25}$}

This experiment was designed to compare responses to Laser$\mathrm{PAS}_{50}$ and to $\mathrm{PAS}_{25}$ protocol in patients with PD, with and without pain, while they were on therapy. A subgroup of 10 patients, 5 with pain in the right upper limb, and 5 patients without pain participated in two separate experimental sessions (Laser-PAS 50 and $\mathrm{PAS}_{25}$ ), randomly. We delivered Laser-PAS 50 as described in Experiment 1, and recorded 20 MEPs before (T0) and after conditioning (T1-T7). PAS $_{25}$ was delivered using peripheral ulnar nerve electric stimulation (cathode proximal) and TMS over the cortical hot-spot for ADM at an interstimulus interval of $25 \mathrm{~ms}$. The intensity of TMS was set to evoke an MEP of $1 \mathrm{mV}$ in the ADM muscle, while the intensity of the ulnar nerve stimulus ( $0.2 \mathrm{~ms}$ duration) was set at 3 times perceptive threshold (by calculating the minimum intensity at which 5 electric stimuli, delivered over the ulnar nerve at the wrist, are all clearly perceived). Two hundred pairs of stimuli were given at $0.25 \mathrm{~Hz}$. MEPs were recorded from the ADM muscle at baseline (T0) and at T1-T7 after PAS 25 , and then averaged.

\section{Statistical analysis}

We used the unpaired Student's t-test to compare the RMT, the intensity to evoke baseline MEPs and for Laser-PAS ${ }_{50}$, heat sensation, the intensity of laser pulse during Laser-PAS ${ }_{50}$, the latency and amplitude of MEP and of main LEP components (N1 and N2/P2), in controls and in patients, when off and on therapy. To assess the effect of dopaminergic therapy on MEP and LEP measurements we used the paired Student's $t$-test. 

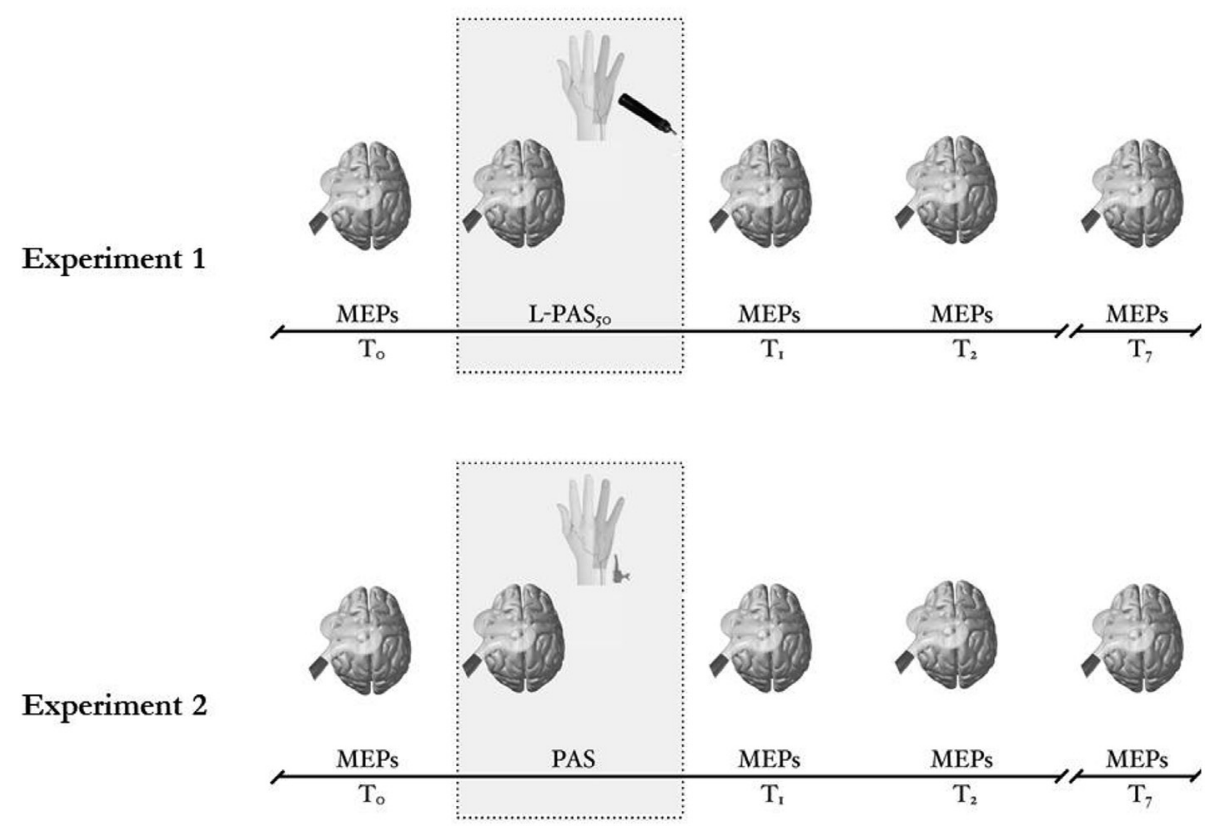

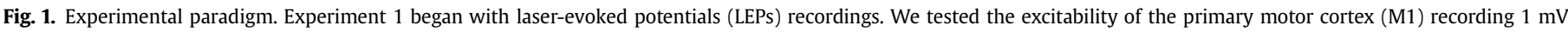

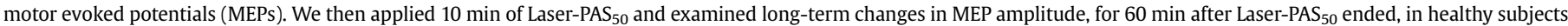

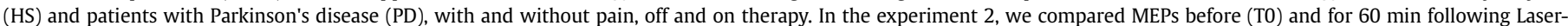

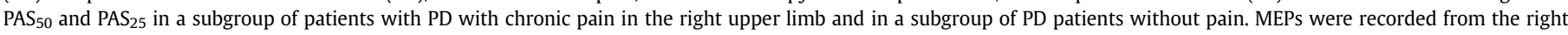
abductor digiti minimi muscle (ADM).

In Experiment 1, to test the effect of Laser-PAS ${ }_{50}$ on MEP amplitude in controls and patients, off and on therapy, we used between-group analysis of variance (ANOVA) with "Group" (healthy subjects vs patients off therapy, healthy subjects vs patients on therapy) and "Time" (T0 vs T1-T7). To clarify the effect of dopaminergic therapy on responses to Laser-PAS ${ }_{50}$ in patients, we also used the two-ways repeated-measures ANOVA with factor "dopaminergic therapy" (off vs on) and factor "Time". In a subsequent analysis, to assess the effect of chronic pain on plasticity induced by Laser-PAS 50 in patients, off and on therapy, we used two separate between-group ANOVAs with factor "Pain" (patients with vs patients without pain, off and on therapy) and "Time". To assess the effect of chronic pain in homotopic and heterotopic body regions in PD, we first compared responses to Laser-PAS 50 in controls and patients with homotopic or heterotopic pain, off and on therapy, by using separate between-group ANOVAs with factor "Group" (healthy subjects vs patients with pain in the right upper limb or patients with pain in other body regions, off and on therapy) and factor "Time". Then, we also used two separate between-group ANOVAs with factor "Pain Localization" (patients with pain in the right upper limb vs patients with pain in other body regions, off and on therapy) and "Time" to better assess the specific effect of chronic pain in homotopic and heterotopic body regions in patients, off and on therapy.

In the Experiment 2, to compare the effect of Laser-PAS 50 and $\mathrm{PAS}_{25}$ on MEP amplitude in patients with chronic pain in the right upper limb, and in patients without chronic pain, on therapy, we used a mixed-design ANOVA with "Group" (patients with pain in the right upper limb vs patients without pain), "Protocol" (Laser$\mathrm{PAS}_{50}$ vs PAS $_{25}$ ) and "Time" (T0 vs T1-T7) as main factors of analysis.

We used the Spearman's R correlation test to assess correlation between patients' clinical features (UPDRS, MMSE, FAB, HAM-D, NRS, disease duration and total L-Dopa equivalent daily doses LEDDs) and changes in MEP amplitude induced by Laser-PAS 50 respect to baseline (T0), at all time intervals. P values less than 0.05 were consider to be statistically significant. The GreenhouseGeisser correction was used when necessary to correct the nonsphericity.

All data are reported as average \pm SD.

\section{Results}

None of the subjects experienced any adverse effects during or after the Laser-PAS ${ }_{50}$. All subjects perceived laser stimuli as painful in all experimental sessions and had LEP values within the normative ranges established in our laboratory [11,26].

In our cohort of patients, 15 out of 20 patients ( 10 men and 5 women, mean age \pm SD: $61.3 \pm 10.4)$ reported at least one type of chronic pain (patient number $2,4-5,7-8,11-20$ ), all of them underwent the DN4 questionnaire and obtained a score $<4$, thus excluding the presence of neuropathic pain (Table 1).

The Student's $t$-test showed comparable RMT, TMS intensity, perceptive threshold, laser pulse intensity, latency and amplitude of MEPs and LEPs, in controls and in patients, when off and on therapy (all p values $>0.05$ ) (Tables 2 and 3 ).

\section{Experiment 1}

ANOVA showed that, after Laser-PAS ${ }_{50}$, MEPs increased in amplitude at T1-T7 in healthy controls, whereas in patients it remained unchanged. The amount of MEP changes induced by Laser-PAS ${ }_{50}$ differed in healthy controls and in patients off therapy. ANOVA showed a significant interaction between factors "Group" and "Time" $\left(\mathrm{F}_{7.266}=6.16 ; \mathrm{p}<0.001\right)$, and a significant effect of factor "Group" $\left(\mathrm{F}_{1.38}=6.13 ; \mathrm{p}=0.02\right)$ and "Time" $\left(\mathrm{F}_{7.266}=8.67\right.$; $\mathrm{p}<0.001$ ). In controls, ANOVA showed a significant effect of the factor "Time" $\left(\mathrm{F}_{7.133}=8.34 ; \mathrm{p}<0.001\right)$; after Laser-PAS ${ }_{50}$, MEP amplitude increased significantly at T2-T7 ( $p<0.01$ for all comparisons). By contrast, in patients off therapy, the factor "Time" was not significant $\left(\mathrm{F}_{7.133}=1.02 ; \mathrm{p}=0.42\right)$. Although MEP amplitude 
was similar at T0 in controls and patients off therapy, after Laser$\mathrm{PAS}_{50}$, MEP amplitude differed significantly at T2-T7 in the two groups ( $\mathrm{p}<0.05$ for all comparisons) (Fig. 2).

Comparing healthy controls and patients on therapy, ANOVA showed significant interaction between factors "Group" and "Time" $\left(\mathrm{F}_{7.266}=4.80 ; \mathrm{p}<0.001\right)$, and a significant effect of factor "Group" $\left(\mathrm{F}_{1.38}=7.10 ; \mathrm{p}=0.01\right)$ and "Time" $\left(\mathrm{F}_{7.266}=9.48 ; \mathrm{p}<0.001\right)$. In controls, ANOVA confirmed a significant effect of the factor "Time" $\left(\mathrm{F}_{7.133}=8.34 ; \mathrm{p}<0.001\right)$; after Laser-PAS ${ }_{50}$, MEP amplitude significantly increased at T2-T7 ( $p<0.01$ for all comparison). By contrast, in patients on therapy, the factor "Time" was not significant $\left(\mathrm{F}_{7.133}=1.50 ; \mathrm{p}=0.17\right)$. Although MEPs were comparable at T0 in healthy subjects and patients on therapy, after Laser-PAS ${ }_{50}$, MEPs differ at T2-T7 ( $p<0.05$ for all comparison) (Fig. 2).

When we assessed the effect of dopaminergic therapy in patients, ANOVA showed no significant effect of the factor "dopaminergic therapy" $\left(\mathrm{F}_{1.19}=0.62 ; \mathrm{p}=0.44\right)$ nor the effect of the factor "Time" $\left(\mathrm{F}_{7.133}=1.26 ; \mathrm{p}=0.22\right)$ (Fig. 2).

When testing the effect of chronic pain on responses to LaserPAS $_{50}$ in patients, off and on therapy, ANOVA showed a non significant effect of the factor "Pain" $\left(\mathrm{F}_{1.18}=0.26 ; \mathrm{p}=0.62\right)$ and "Time" $\left(\mathrm{F}_{7.126}=0.63 ; \mathrm{p}=0.73\right)$ when off and on state of therapy $\left(\mathrm{F}_{1.18}=0.22 ; \mathrm{p}=0.65\right.$ and $\mathrm{F}_{7.126}=1.24 ; \mathrm{p}=0.29$, respectively $)$ (Fig. 3).

Comparing controls with patients with pain in the right upper $\operatorname{limb}(\mathrm{n}=10$, patient number $4,7,8,11,12$ and 16-20), ANOVA showed a significant interaction between factors "Group" and "Time", when off and on therapy $\left(\mathrm{F}_{7.196}=3.01 ; \mathrm{p}<0.01\right.$ and $\mathrm{F}_{7.196}=4.07 ; \mathrm{p}<0.01$, respectively) and a significant effect of factor "Group" $\left(\mathrm{F}_{1.28}=4.22 ; \mathrm{p}<0.01\right.$ and $\mathrm{F}_{1.28}=6.38 ; \mathrm{p}<0.01$, respectively $)$ and "Time" $\left(\mathrm{F}_{7.196}=5.07 ; \mathrm{p}<0.01\right.$ and $\mathrm{F}_{7.196}=3.58 ; \mathrm{p}<0.01$, respectively). Post hoc analysis showed that MEPs increased in amplitude at T3-T7 (all p values $<0.05$ ) in controls but not in patients with pain in the right upper limb, when off and on therapy. Differently, when we tested responses to Laser-PAS 50 in controls and patients with pain in other body regions $(n=5$, patient number $2,5,13,14,15)$, off and on therapy, ANOVA showed a significant effect of the factor "Time" $\left(\mathrm{F}_{7.16}=4.80 ; \mathrm{p}<0.01\right.$ and $\mathrm{F}_{7.16}=4.53$; $\mathrm{p}<0.01$, respectively), whereas factor "Group" $\left(\mathrm{F}_{1.23}=0.46 ; \mathrm{p}=0.5\right.$
Table 3

Transcranial magnetic stimulation (TMS) data in the experiment testing primary motor cortex (M1) plasticity in 20 healthy subjects (HS) and in 20 patients with Parkinson's disease (PD), off and on therapy. RMT = Resting motor threshold; Int. for MEPs = Intensity for $1 \mathrm{mv}$ MEP.

\begin{tabular}{lll}
\hline & RMT (\%) & Int. for MEPs (\%) \\
\hline Controls & $41.1 \pm 4.9$ & $54.7 \pm 9.4$ \\
PD off & $41.3 \pm 15.2$ & $54.3 \pm 20.2$ \\
PD on & $43.9 \pm 15$ & $57.2 \pm 17.4$ \\
\hline
\end{tabular}

and $\mathrm{F}_{1.23}=0.67 ; \mathrm{p}=0.42$, respectively) and the interaction between "Group" and "Time" $\left(\mathrm{F}_{7.161}=0.49 ; \mathrm{p}=0.84\right.$ and $\mathrm{F}_{7.161}=0.52$; $\mathrm{p}=0.82$, respectively) were both non significant.

When comparing responses to Laser-PAS 50 in patients, off and on therapy, with pain in the right upper limb with patients with pain in other body regions, ANOVA showed a significant interaction between factors "Pain Localization" and "Time" ( $F_{7.91}=3.33$; $\mathrm{p}<0.01$ ) and a significant effect of the factor "Pain Localization" $\left(\mathrm{F}_{1.13}=4.68 ; \mathrm{p}=0.04\right)$ and "Time" $\left(\mathrm{F}_{7.91}=6.46 ; \mathrm{p}<0.01\right)$ in patients off therapy. Post hoc analysis showed that MEPs decreased in amplitude at T3-T5 (all p values $<0.05$ ) in patients with pain in the right upper limb compared with patients with pain in other body regions. Similarly, in patients on therapy ANOVA showed a significant interaction between factors "Pain Localization" and "Time" $\left(\mathrm{F}_{7.91}=2.39 ; \mathrm{p}=0.03\right)$ and a significant effect of the factor "Pain Localization" $\left(\mathrm{F}_{1.13}=7.64 ; \mathrm{p}=0.02\right)$ and "Time" $\left(\mathrm{F}_{7.91}=2.20\right.$; $\mathrm{p}=0.04)$. Post hoc analysis showed that MEPs decreased in amplitude at T4-T7 (all p values $<0.05$ ) in patients with pain in the right upper limb compared with patients with pain in other body regions (Fig. 4).

\section{Experiment 2}

In the Experiment 2, ANOVA showed a significant effect of the factor "Protocol" $\left(\mathrm{F}_{1.8}=8.48 ; \mathrm{p}=0.02\right)$ and "Time" $\left(\mathrm{F}_{7.56}=3.61\right.$; $\mathrm{p}<0.01$ ). In patients with pain in the right upper limb, post hoc analysis showed an interaction between the factors "Protocol" and "Time" $\left(\mathrm{F}_{7.28}=4.07 ; \mathrm{p}<0.01\right)$ and a significant effect of the factor "Protocol" $\left(\mathrm{F}_{1.4}=139.98 ; \mathrm{p}<0.01\right)$ and "Time" $\left(\mathrm{F}_{7.28}=4.40\right.$;

Table 2

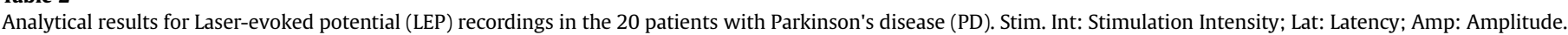

\begin{tabular}{|c|c|c|c|c|c|c|c|c|}
\hline Patients & $\frac{\text { Perceptive }}{\text { Threshold }\left(\mathrm{mJ} / \mathrm{mm}^{2}\right)}$ & $\frac{\text { Stim Int. }}{\left(\mathrm{mJ} / \mathrm{mm}^{2}\right)}$ & $\frac{\mathrm{N} 1 \text { lat. }}{(\mathrm{ms})}$ & $\frac{\mathrm{N} 1 \mathrm{amp} .}{(\mu \mathrm{V})}$ & $\frac{\mathrm{N} 2 \text { lat. }}{(\mathrm{ms})}$ & $\frac{\mathrm{N} 2 \mathrm{amp} .}{(\mu \mathrm{V})}$ & $\frac{\text { P2 lat. }}{(\mathrm{ms})}$ & $\frac{\text { P2 amp. }}{(\mu \mathrm{V})}$ \\
\hline 1 & 51 & 102 & 188 & 8.6 & 210 & 9.2 & 314 & 13.2 \\
\hline 2 & 51 & 127 & 200 & 5.0 & 240 & 4.0 & 350 & 8.0 \\
\hline 3 & 70 & 153 & 198 & 3.0 & 236 & 7.0 & 438 & 2.0 \\
\hline 4 & 76 & 153 & 220 & 6.5 & 284 & 4.5 & 420 & 16.0 \\
\hline 5 & 38 & 102 & 152 & 6.0 & 194 & 16.0 & 330 & 14.0 \\
\hline 6 & 64 & 127 & 182 & 3.0 & 216 & 4.0 & 298 & 20.0 \\
\hline 7 & 39 & 115 & 220 & 6.0 & 248 & 10.0 & 350 & 18.0 \\
\hline 8 & 51 & 127 & 188 & 10.0 & 239 & 7.2 & 360 & 13.0 \\
\hline 9 & 50 & 127 & 216 & 6.0 & 244 & 6.0 & 318 & 1.5 \\
\hline 10 & 50 & 114 & 218 & 13.0 & 252 & 7.0 & 366 & 6.0 \\
\hline 11 & 38 & 127 & 220 & 5.0 & 240 & 10.0 & 395 & 7.5 \\
\hline 12 & 51 & 127 & 138 & 9.0 & 180 & 8.0 & 300 & 12.0 \\
\hline 13 & 50 & 127 & 198 & 6.5 & 238 & 13.0 & 358 & 7.0 \\
\hline 14 & 51 & 127 & 188 & 6.0 & 230 & 3.0 & 294 & 9.0 \\
\hline 15 & 40 & 140 & 244 & 5.0 & 246 & 8.4 & 390 & 7.0 \\
\hline 16 & 38 & 102 & 186 & 5.0 & 244 & 1.0 & 330 & 17.0 \\
\hline 17 & 38 & 127 & 164 & 1.0 & 223 & 6.0 & 356 & 14.0 \\
\hline 18 & 25 & 178 & 230 & 4.3 & 274 & 4.3 & 384 & 6.0 \\
\hline 19 & 76 & 153 & 178 & 13.2 & 226 & 9.6 & 318 & 22.6 \\
\hline 20 & 51 & 127 & 193 & 6.5 & 230 & 7.0 & 350 & 9.0 \\
\hline Av & 49.9 & 129.1 & 195.05 & 6.4 & 234.7 & 7.26 & 350.95 & 11.1 \\
\hline SD & 13.3 & 19.1 & 26.4 & 3.1 & 23.7 & 3.5 & 41.0 & 5.8 \\
\hline
\end{tabular}




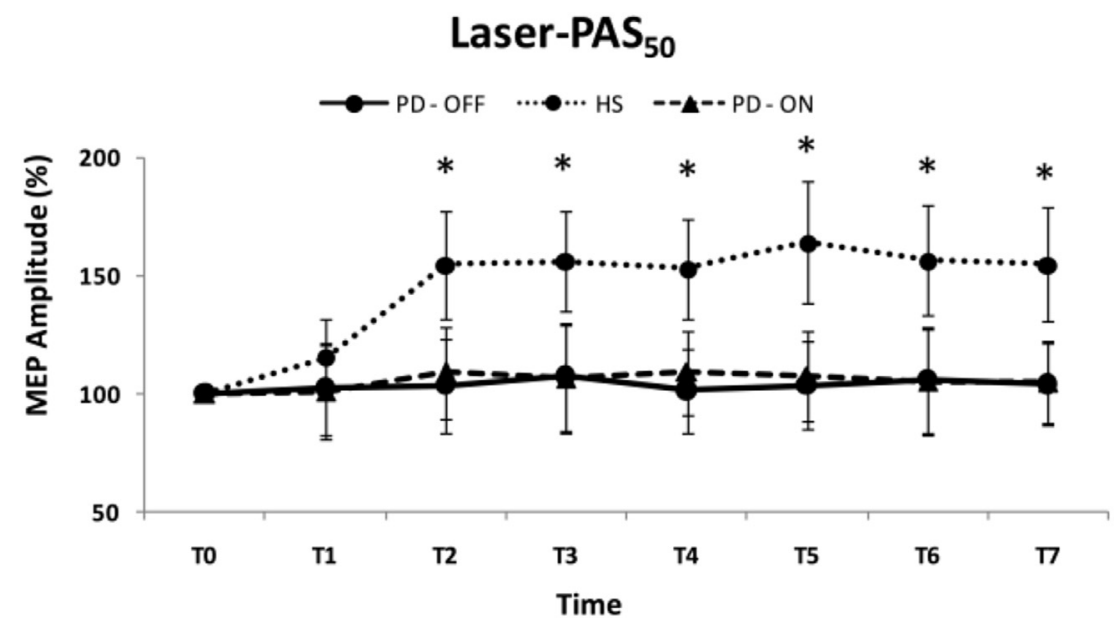

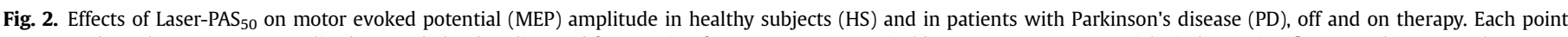

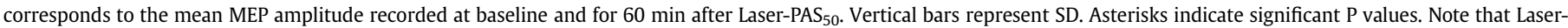
$\mathrm{PAS}_{50}$ increased MEP amplitude in healthy subjects but not in patients with PD, off and on therapy.

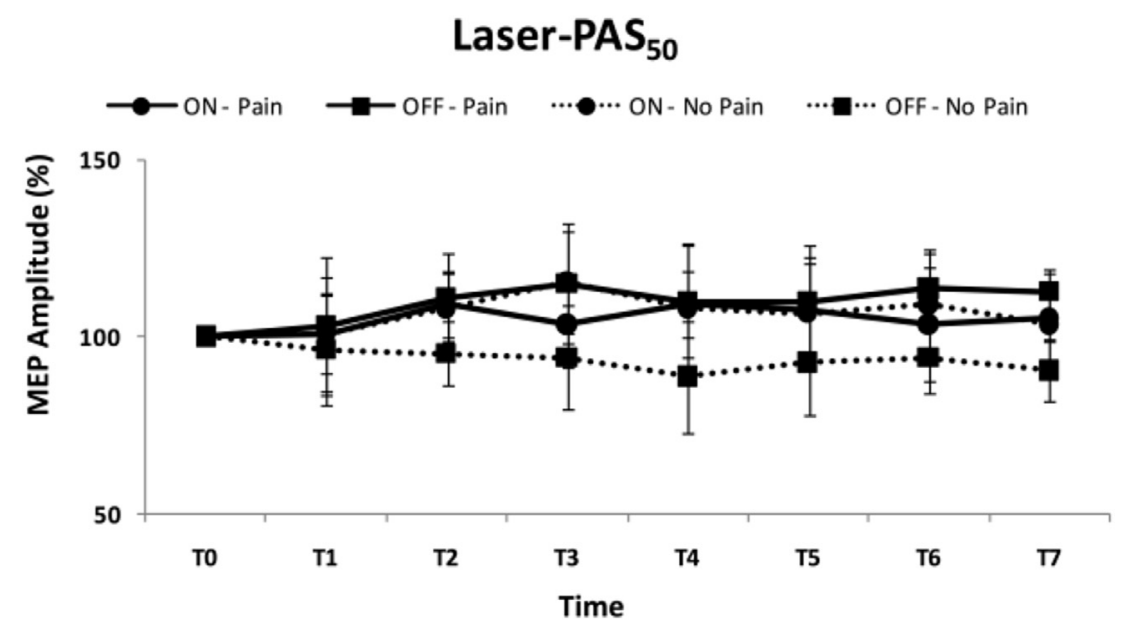

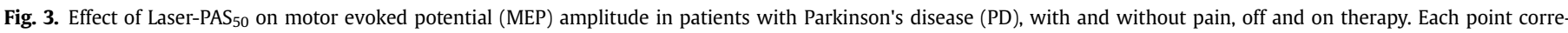

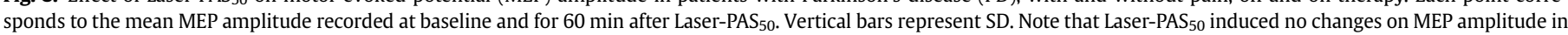
PD patient, with and without pain, off and on therapy.

$\mathrm{p}<0.01)$. Although in patients with pain in the right upper limb, MEPs were comparable before Laser-PAS 50 and $\operatorname{PAS}_{25}(\mathrm{TO})(\mathrm{p}=1)$, compared to $\mathrm{PAS}_{25}$, Laser-PAS 50 elicited lower amplitude MEPs at T2-T7 (all p values $<0.05$ ). Conversely, in patients without pain, post hoc analysis showed a non significant effect of the factors "Protocol" $\left(\mathrm{F}_{1.4}=0.81 ; \mathrm{p}=0.42\right)$ and "Time" $\left(\mathrm{F}_{7.28}=1.58 ; \mathrm{p}=0.18\right)$ demonstrating that MEPs were comparable before and after LaserPAS $_{50}$ and PAS $_{25}$ (all p > 0.05) (Fig. 5).

The Spearman's R correlation test found no correlation between patients' clinical features at all the time intervals. In contrast, Spearman's correlation test showed a significant negative correlation between the severity of pain reported in the right upper limb, as assessed by the NRS, and the percentage of MEP amplitude increase at $\mathrm{T} 5$ after Laser-PAS 50 in patients off $(\mathrm{R}=-0.78$ and $\mathrm{p}=0.01)$ and on therapy $(\mathrm{R}=-0.70$ and $\mathrm{p}=0.02)$ (Fig. $6 \mathrm{~A}$ and $\mathrm{B})$.

\section{Discussion}

In this study, we demonstrated abnormal MEP responses to Laser-PAS $_{50}$ in PD. Dopaminergic therapy left responses to Laser$\mathrm{PAS}_{50}$ globally unchanged. The response to Laser-PAS 50 was comparable in patients with and without chronic pain. In patients with pain in the limb directly investigated with Laser-PAS 50 , we found prominent abnormal responses than in patients with pain in other body regions. We provide here the first evidence of a reduced responses to Laser-PAS 50 in patients with and without chronic pain, off and on therapy, reflecting an altered pain-motor integration.

When testing the effects of Laser-PAS 50 in PD, we have excluded a number of methodological factors possibly leading to misinterpretation of our data. Given that we found comparable RMT values, intensity to evoke MEPs of $1 \mathrm{mV}$ amplitude and intensity for TMS during Laser-PAS ${ }_{50}$, in healthy controls and patients, off and on therapy, we excluded possible confoundings due to altered integrity of the cortico-spinal tract as well as difference in M1 baseline excitability. We also used comparable laser intensity during Laser$\mathrm{PAS}_{50}$, in controls and patients, off and on therapy. During each experimental session, we checked the patients' muscle relaxation and none of the patients showed voluntary or involuntary muscular activity before, during or after Laser-PAS 50 excluding homeostatic or non-homeostatic interaction between muscular activity and TMS [27-31]. In this study, when we applied repeated sessions of Laser$\mathrm{PAS}_{50}$ in the same patient, we used between-session intervals of at 


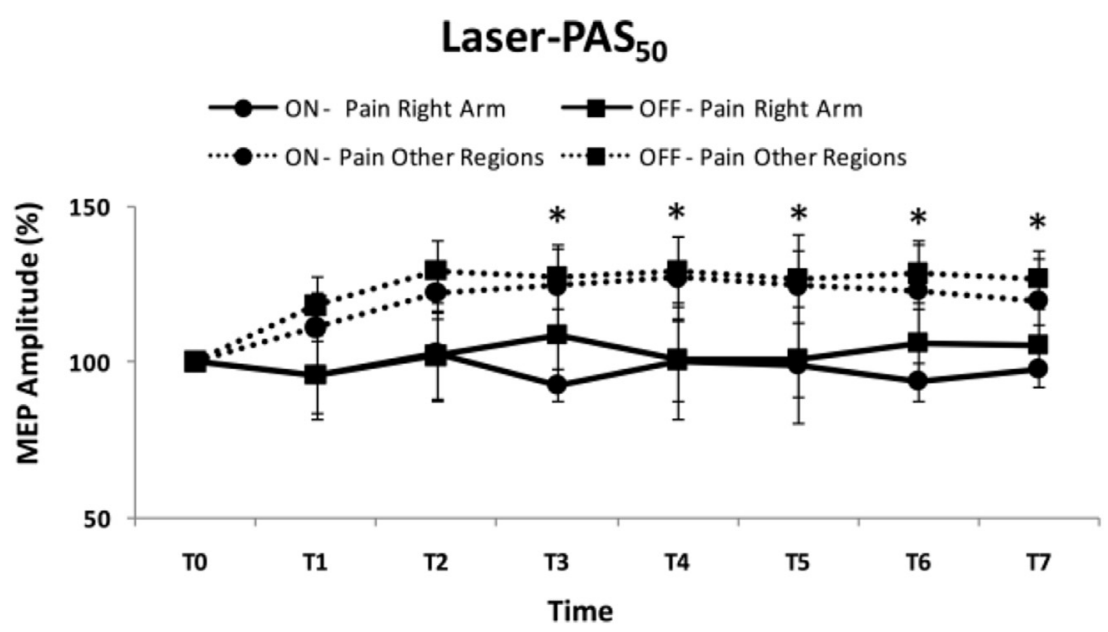

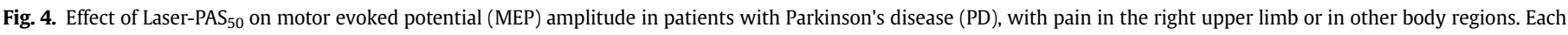

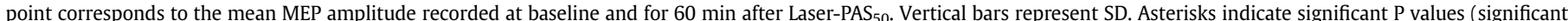

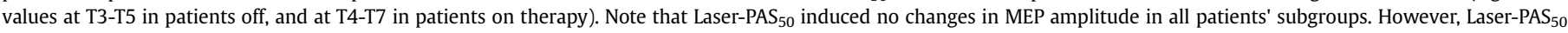
induced lower responses in patients with pain in the right upper limb than those observed in patients with pain in other body regions, off and on therapy.

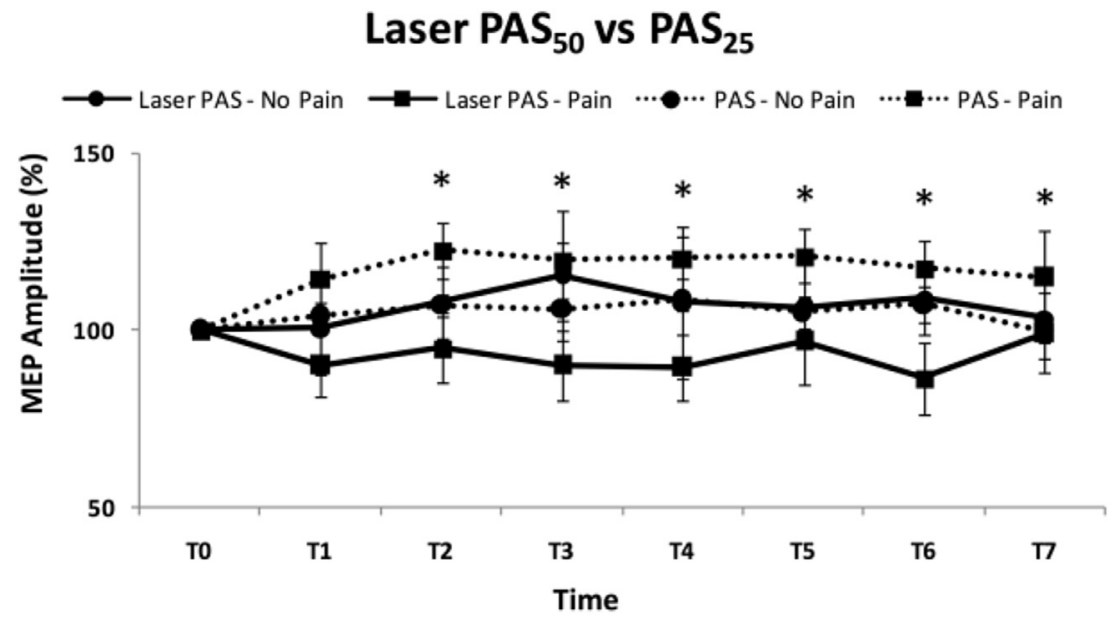

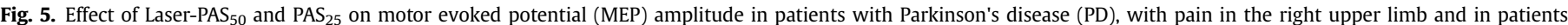

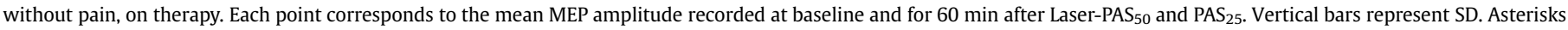

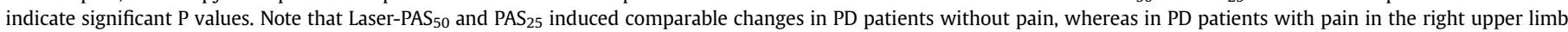

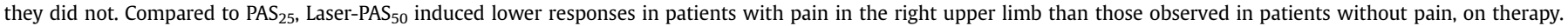

least one week making highly unlikely the possible interference between the two experimental sessions [28,31,32].

\section{Laser-PAS 50 elicits reduced responses in Parkinson's disease}

The reduced responses to Laser-PAS 50 we observed in patients off therapy, may reflect a number of pathophysiological mechanisms. We can reasonably exclude a significant contribution of small-nerve fibre damage, previously demonstrated in a skin biopsy study in patients with PD [33] since none of the participants manifested or referred any dysautonomic symptom and none had pure neuropathic pain. Moreover, we found comparable laser perceptive thresholds, latency and amplitude of N1- and N2/P2-LEP components in healthy controls and in patients, with and without pain. Similar laser perceptive thresholds and latency/amplitude of the N1 and N2/P2-LEP components in healthy controls and in patients, with and without pain, also exclude damage of peripheral and central nociceptive afferent pathways. These data therefore indicate that our patients with pain did not suffer from neuropathic pain [34]. The observation of comparable LEP amplitudes in controls and in patients with PD argues against two previous laser studies, that using a $\mathrm{CO}_{2}$ laser stimulator reported abnormal LEPs in PD. Schestatsky et al. [14] found an increased LEP amplitude in patients with PD with neurophatic (central) pain; conversely, Tinazzi et al. [15] stimulating the shoulder, reported a decreased LEP amplitude in patients with nociceptive (muscoloskeletal) pain. In our study, however, we used a different laser stimulator, investigated the hand and none of our patients suffered from neurophatic (central) pain, as assessed with DN4. Hence, the hypothesis that possible changes in N2/P2 LEP component influenced our findings is very unlikely. A more likely hypothesis implies that the reduced responses to Laser-PAS 50 we observed in patients, off therapy, reflects changes in M1 plasticity mechanisms. In a previous study in healthy subjects [16], we found that, Laser-PAS ${ }_{50}$ induces MEP amplitude changes that might reflect spike-timingdependent plasticity (STDP) in M1 [16,17]. STDP in M1 is supposed to reflect the coincidence detection of excitatory postsynaptic potentials in the apical dendrites (layer 2/3) and back- 


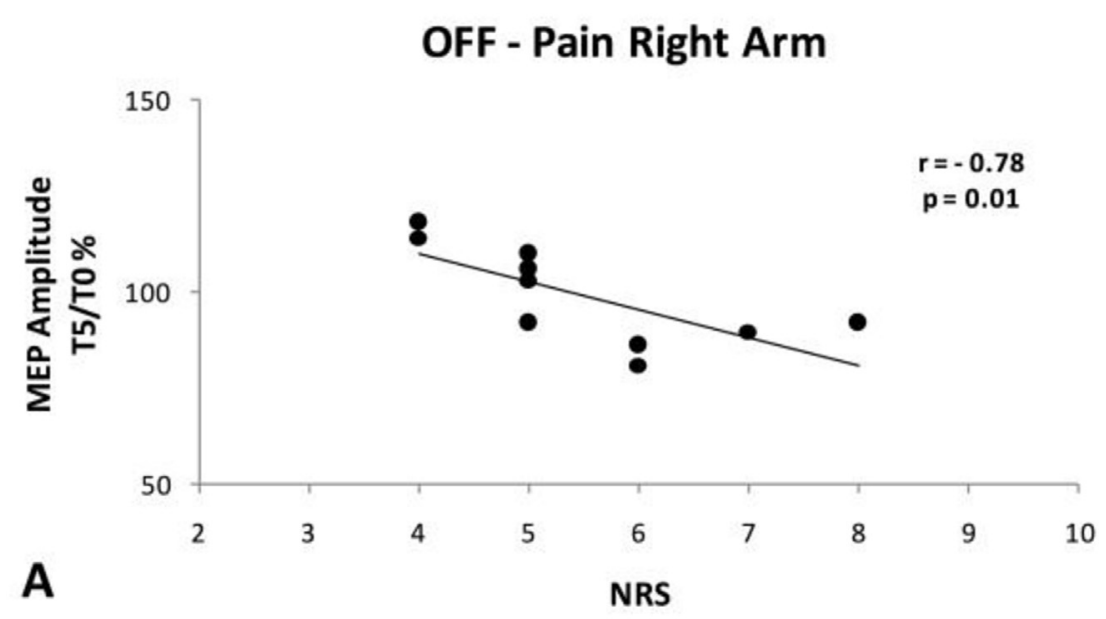

ON - Pain Right Arm

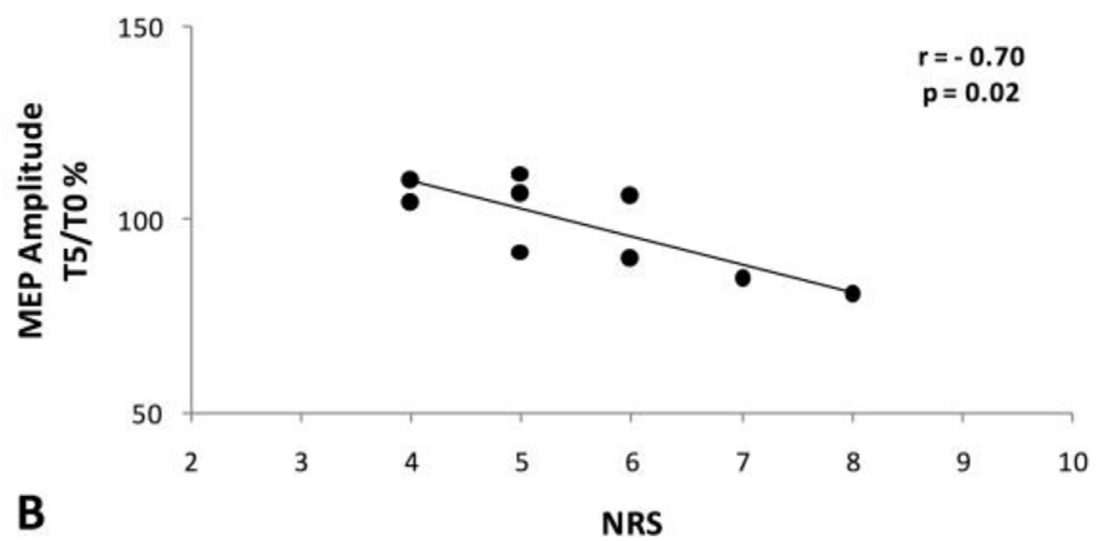

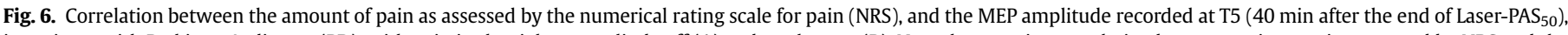

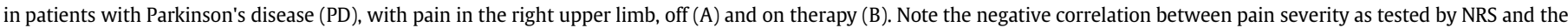
amount of MEP amplitude changes following Laser-PAS ${ }_{50}$ in patients with PD off (A) and on therapy (B).

propagating action potentials from layer 5 neurons [16,35-38]. Since TMS activates layer 5 cortical-spinal neurons [39,40] and cortical projections originating from remote cortical pain-related areas reach $\mathrm{M} 1$ in cortical layers $2 / 3[41-43]$, it is likely that Laser-PAS $_{50}$ induces STDP in cortical layer 2/3 of M1. Hence, the hypothesis we favor is that in PD patients off therapy, the reduced response to Laser-PAS 50 reflects abnormal STDP in M1.

\section{Dopaminergic therapy does not restore responses to Laser-PAS 50}

We found that L-Dopa left responses to Laser-PAS ${ }_{50}$ unchanged in patients with and without chronic pain, suggesting that dopaminergic therapy does not restore abnormal STDP in M1 in PD, in line with previous observations with other types of plasticityinducing protocols [16,44-48]. Our findings indirectly support the hypothesis that non-dopaminergic pathways contribute to the pathophysiology of pain in PD [2,4]. Extranigral pathology in PD, including neurodegeneration in the noradrenergic, serotonergic, and cholinergic systems, contributes to pain modulation $[7,8,49,50]$. The hypothesis that a non-dopaminergic pathway contributes to the pathophysiology of chronic pain in PD is also in line with the observation that dopaminergic therapy only partly improve pain $[2,4]$.

The response to Laser-PAS 50 differs according to pain localization in Parkinson's disease

When comparing patients with chronic pain (regardless of the specific body region affected by pain) and patients without pain, we found comparable responses to Laser-PAS $\mathrm{S}_{50}$ in the two groups. This observation would suggest that in $\mathrm{PD}$, chronic pain does not influence per se the response to Laser-PAS 50 . When we compared however, patients with pain in the right upper limb and patients with pain in other body regions (but not in the right upper limb), we found different responses to Laser-PAS 50 in the two patients' subgroup: patients with pain in the right upper limb had prominent alterations in responses to Laser-PAS ${ }_{50}$, whereas patients with pain in other body regions were characterized by less severe neurophysiological abnormalities. It should be noted, that in our analysis, we found different responses to Laser-PAS ${ }_{50}$ when comparing controls and patients with homotopic but not heterotopic pain probably due to the low number of subjects analysed in the third group (controls: $n=20$; patients with homotopic pain: 
$\mathrm{n}=10$; patients with heterotopic pain: $\mathrm{n}=5$ ). Interestingly, we found a negative correlation between the severity of pain in the right upper limb, as tested by NRS scores, and the amount of response to Laser-PAS 50 . Given that patients with pain in the right upper limb had similar clinical features including MDS-UPDRS motor scores compared to those with pain in other body regions, we can exclude that the prominent abnormalities in MEP changes observed after Laser-PAS 50 , in patients with pain in the right upper limb, merely arised from more severe motor symptoms. Overall our findings demonstrate that in patients with chronic pain, the severity of M1 STDP abnormalities is prominent when Laser-PAS 50 examines the body region specifically affected by pain further suggesting abnormal pain-motor integration in PD.

\section{Pain-motor and sensori-motor integration in Parkinson's disease}

The reduced responses to Laser-PAS 50 might arise from abnormal STDP in M1 that does not strictly depend on the specific PAS protocol used. Previous studies with the original $\mathrm{PAS}_{25}$ protocol have demonstrated abnormal responses to $\mathrm{PAS}_{25}$ in PD [45,48,51-59]. In more detail, the response to $\mathrm{PAS}_{25}$ has been consistently found to be reduced in PD following $\mathrm{PAS}_{25}$ over the most affected M1, as in our study. According to the hypothesis that the reduced responses to Laser-PAS 50 arise from abnormal M1 STDP not strictly depending on the specific PAS protocol used, patients with PD, with and without pain, should have manifested comparable responses to Laser-PAS 50 and PAS $_{25}$, since both PAS protocols are known to elicit STDP in M1. In this study, however, this was not the case. In our second set of experiments, we found comparable responses to Laser-PAS 50 and PAS $_{25}$ only in patients without pain. By contrast, in patients with homotopic pain, Laser-PAS 50 elicited greater abnormalities than $\mathrm{PAS}_{25}$. Previous studies have shown that chronic musculoskeletal pain may lead to cortical reorganization processes in M1 muscle representation [60-63]. Accordingly, it might be argued that in PD, pain-induced cortical reorganization contributed to the differential response to Laser-PAS 50 and $\mathrm{PAS}_{25}$ we observed in patients with homotopic pain. In this study, we did not examine and compare cortical maps of ADM muscles in patients with and without pain and thus we cannot fully exclude that a painrelated cortical map reorganization might have contributed at least in part to our findings. However, this hypothesis seems unlikely since in PD patients with homotopic pain, a pain-induced cortical reorganization would have similarly affected responses to both Laser-PAS 50 and $\mathrm{PAS}_{25}$ protocols. We therefore suggest that the reduced responses to Laser-PAS 50 in patients with homotopic pain reflect abnormal M1 SDTP specifically induced by the activation of the nociceptive system. Hence, we suggest that in PD, and specifically in patients with chronic pain, the reduced responses to Laser$\mathrm{PAS}_{50}$ reflect abnormal pain-motor integration processes in functionally connected brain regions involved in pain matrices. In the present study, we did not compared pain-motor integration and sensori-motor integration in patients with chronic pain in other body regions because this subgroup of patients was characterized by heterogeneous pain localization, thus precluding a precise evaluation of pain-motor integration processes. Accordingly, the possible effect of heterotopic pain on pain-motor integration processes in patients with PD remains to be clarified.

\section{Brain networks underlying abnormal pain-motor integration in Parkinson's disease}

In a previous study in healthy subjects [16], we have suggested that Laser-PAS 50 elicits STDP in M1 through mechanisms of painmotor integration reflecting the functional connectivity between pain and motor areas involved in the pain matrix (ACC, insula, S2, supplementary motor area and M1). The pain matrix is thought to be responsible for cortical processing and conscious experience of pain [12,64-67]. Experimental evidence in animals and also in humans have demonstrated that most of the cortical areas contributing to the pain matrix are also targeted by dopaminergic projections including the "mesolimbic" and "mesocortical" dopaminergic pathways and that those pathways play a role in nociception $[68,69]$. Previous studies in healthy subjects with positron emission tomography have demonstrated increased dopaminergic release following various types of experimental pain [70-74]. In patients with $\mathrm{PD}$, a study with functional magnetic resonance imaging demonstrated increased pain-induced activation of the right insula, prefrontal and left ACC in patients off therapy, but reduced activation in the same cortical areas in the on state of therapy, compared with controls [75]. Overall these findings support the hypothesis that in PD, altered mesolimbic and mesocortical dopaminergic pathways influence the activation of the pain matrix possibly leading to abnormal cortical pain-motor integration. Whether one specific cortical area, among those contributing to the pain matrix is responsible of abnormal cortical pain-motor integration in $\mathrm{PD}$, remains to be clarified. It remains also unclear whether abnormal cortical pain-motor integration might predispose or contribute to the pathophysiology of chronic pain in PD.

A further comment concerns the possibility that in PD, abnormal pain-motor integration may occur also in subcortical brain regions. Experimental studies in animals and in humans have demonstrated that dopamine exerts a key role in modulating the "brainstem descending pain-modulatory system" [70-74,76]. Hence, in PD, dopaminergic degeneration in the substantia nigra pars compacta might drive an altered activation of the "brainstem descending pain-modulatory system" leading to abnormal pain-motor integration in the spinal cord. Furthermore, in PD, abnormal pain motor integration might occur also at basal ganglia level since dopaminergic denervation changes the release of neuropeptides contributing to nociception. It is known that striatal medium spiny neurons that give rise to the "direct pathway" release substance $\mathrm{P}$ and dynorphin, whereas those involved in the "indirect pathway" release enkephalin [77-79]. Experimental studies in animals have demonstrated that neurons in the substantia nigra, caudate, putamen and globus pallidus clearly respond to noxious stimulation $[9,80,81]$. In addition, the basal ganglia receive nociceptive information also directly from the cortex, thalamus and amygdala $[9,80,81]$. The laser-PAS 50 technique however, has been designed to examine specifically cortical pain-motor integration processes. Future studies would clarify the possible pathophysiological role of abnormal pain-motor integration processes at brainstem and basal ganglia level.

When interpreting our neurophysiological observations in PD, with and without chronic pain, several limitations should be taken into account. Given that we recorded LEPs in all patients on therapy, we cannot exclude a possible effect of dopaminergic therapy on N1 and N2/P2 LEP components in patients with and without pain. This hypothesis however, seems unlikely as suggested by a previous LEP study [82]. Future studies however, will clarify possible changes in pain-motor integration in drug naïve patients with $\mathrm{PD}$, thus excluding possible confounding factors secondary to dopaminergic treatments. In our cohort of patients with PD, we studied no patients with a neuropathic pain including the central parkinsonian pain [4]. Hence, we cannot clarify whether the response to LaserPAS $_{50}$ may differ in patients with nociceptive or neuropathic pain. Future studies will clarify whether the nociceptive or neuropathic pain component predominantly contribute to the abnormal response to Laser-PAS 50 in PD. 


\section{Conclusions}

In this study, we report abnormal responses to Laser-PAS 50 in patients with PD, and prominently in those with chronic pain, which point to abnormal pain-motor integration. In addition, given that a number of experimental and clinical studies have demonstrated that invasive as well as non-invasive motor cortex stimulation induce significant pain relief in patients with pain [83-87], our findings provide further information for designing new therapeutic strategies with non-invasive brain stimulating protocols for treating chronic pain in PD.

\section{Conflict of interest}

None.

\section{Acknowledgements}

We have no acknowledgements to declare.

\section{References}

[1] Defazio G, Berardelli A, Fabbrini G, Martino D, Fincati E, Fiaschi A, et al. Pain as a nonmotor symptom of Parkinson's disease. Arch Neurol 2008;65:1191-4.

[2] Defazio G, Tinazzi M, Berardelli A. How pain arises in Parkinson's disease? Eur J Neurol 2013;20:1517-23.

[3] Broen MP, Braaksma MM, Patijn J, Weber WE. Prevalence of pain in Parkinson's disease: a systematic review using the modified QUADAS tool. Mov Disord 2012:27:480-4.

[4] Cury RG, Galhardoni R, Fonoff ET, Perez Lloret S, Dos Santos Ghilardi MG, Barbosa ER, et al. Sensory abnormalities and pain in Parkinson disease and its modulation by treatment of motor symptoms. Eur J Pain 2015;20:151-65.

[5] Wasner G, Deuschl G. Pains in Parkinson disease-many syndromes under one umbrella. Nat Rev Neurol 2012;17:284-94.

[6] Truini A, Frontoni M, Cruccu G. Parkinson's disease related pain: a review of recent findings. J Neurol 2013;260:330-4.

[7] Braak H, Del Tredici K, Bratzke H, Hamm-Clement J, Sandmann-Keil D, Rüb U. Staging of the intracerebral inclusion body pathology associated with idiopathic Parkinson's disease (preclinical and clinical stages). J Neurol 2002;249; $1-5$.

[8] Gold A, Turkalp ZT, Munoz DG. Enteric alpha-synuclein expression is increased in Parkinson's disease but not Alzheimer's disease. Mov Disord 2013:28:237-40.

[9] Conte A, Khan N, Defazio G, Rothwell JC, Berardelli A. Pathophysiology of somatosensory abnormalities in Parkinson disease. Nat Rev Neurol 2013;9: 687-97.

[10] Perchet C, Godinho F, Mazza S, Frot M, Legrain V, Magnin M, et al. Evoked potentials to nociceptive stimuli delivered by CO2 or Nd:YAP lasers. Clin Neurophysiol 2008;119:2615-22.

[11] Cruccu G, Aminoff MJ, Curio G, Guerit JM, Kakigi R, Mauguiere F, et al. Recommendations for the clinical use of somatosensory-evoked potentials. Clin Neurophysiol 2008;119:1705-19.

[12] Garcia-Larrea L, Frot M, Valeriani M. Brain generators of laser evoked potentials: from dipoles to functional significance. Neurophysiol Clin 2003;33: 279-92.

[13] Iannetti GD, Mouraux A. From the neuromatrix to the pain matrix (and back). Exp Brain Res 2010;205:1-12.

[14] Schestatsky P, Kumru H, Valls-Solé J, Valldeoriola F, Marti MJ, Tolosa E, et al. Neurophysiologic study of central pain in patients with Parkinson disease. Neurology 2007;69:2162-9.

[15] Tinazzi M, Recchia S, Simonetto S, Tamburin S, Defazio G, Fiaschi A, et al. Muscular pain in Parkinson's disease and nociceptive processing assessed with CO2 laser evoked potentials. Mov Disord 2010;25:213-20.

[16] Suppa A, Biasiotta A, Belvisi D, Marsili L, La Cesa S, Truini A, et al. Heat-evoked experimental pain induces long-term potentiation-like plasticity in human primary motor cortex. Cereb Cortex 2013;23:1942-51.

[17] Stefan K, Kunesch E, Cohen LG, Benecke R, Classen J. Induction of plasticity in the human motor cortex by paired associative stimulation. Brain 2000;123: 572-84.

[18] Gibb WR, Lees AJ. The relevance of the Lewy body to the pathogenesis of idiopathic Parkinson's disease. J Neurol Neurosurg Psychiatry 1988;51: $745-52$.

[19] Berardelli A, Wenning GK, Antonini A, Berg D, Bloem BR, Bonifati V, et al. EFNS/MDS-ES/ENS [corrected] recommendations for the diagnosis of Parkinson's disease. Eur J Neurol 2013;20:16-34.

[20] Goetz CG, Tilley BC, Shaftman SR, Stebbins GT, Fahn S, Martinez-Martin P, et al. Movement disorder society-sponsored revision of the unified
Parkinson's disease rating scale (MDS-UPDRS): scale presentation and clinimetric testing results. Mov Disord 2008;23:2129-70.

[21] Antonini A, Abbruzzese G, Ferini-Strambi L, Tilley B, Huang J, Stebbins GT, et al. Validation of the Italian version of the movement disorder society- unified Parkinson's disease rating scale. Neurol Sci 2013;34:683-7.

[22] Folstein MF, Folstein SE, McHugh PR. "Mini-mental state". A practical methoc for grading the cognitive state of patients for the clinician. J Psychiatr Res 1975;12:189-98.

[23] Dubois B, Slachevsky A, Litvan I, Pillon B. The FAB: a frontal assessment Battery at bedside. Neurology 2000;55:1621-6.

[24] Hamilton M, Guy W. Hamilton depression scale. Berlin: Beltz; 1976.

[25] Bouhassira D, Attal N, Alchaar H, Boureau F, Brochet B, Bruxelle J, et al. Comparison of pain syndromes associated with nervous or somatic lesions and development of a new neuropathic pain diagnostic questionnaire (DN4) Pain 2005;114:29-36.

[26] Truini A, Piroso S, Pasquale E, Notartomaso S, Di Stefano G, Lattanzi R, et al. Nacetyl-cysteine, a drug that enhances the endogenous activation of group-Il metabotropic glutamate receptors, inhibits nociceptive transmission in humans. Mol Pain 2015;20:11-4.

[27] Huang YZ, Rothwell JC, Edwards MJ, Chen RS. Effect of physiological activity on an NMDA-dependent form of cortical plasticity in human. Cereb Cortex 2008; $18: 563-70$.

[28] Huang YZ, Rothwell JC, Chen RS, Lu CS, Chuang WL. The theoretical model of theta burst form of repetitive transcranial magnetic stimulation. Clin Neurophysiol 2010;122:1011-8.

[29] Gentner R, Wankerl K, Reinsberger C, Zeller D, Classen J. Depression of human corticospinal excitability induced by magnetic theta-burst stimulation: evidence of rapid polarity-reversing metaplasticity. Cereb Cortex 2008;18: 2046-53.

[30] Iezzi E, Conte A, Suppa A, Agostino R, Dinapoli L, Scontrini A, et al. Phasic voluntary movements reverse the aftereffects of subsequent theta-burst stimulation in humans. J Neurophysiol 2008;100:2070-6.

[31] Siebner HR, Hartwigsen G, Kassuba T, Rothwell JC. How does transcranial magnetic stimulation modify neuronal activity in the brain? Implications for studies of cognition. Cortex 2009;45:1035-42.

[32] Todd G, Flavel SC, Ridding MC. Priming theta-burst repetitive transcrania magnetic stimulation with low- and high-frequency stimulation. Exp Brain Res 2009;195:307-15.

[33] Nolano M, Provitera V, Estraneo A, Selim MM, Caporaso G, Stancanelli A, et al Sensory deficit in Parkinson's disease: evidence of a cutaneous denervation. Brain 2008;131:1903-11.

[34] Treede RD, Jensen TS, Campbell JN, Cruccu G, Dostrovsky JO, Griffin JW, et al. Neuropathic pain: redefinition and a grading system for clinical and research purposes. Neurology 2008;70:1630-5.

[35] Dan Y, Poo MM. Spike timing-dependent plasticity of neural circuits. Neuron 2004; $44: 23-30$.

[36] Dan Y, Poo MM. Spike timing-dependent plasticity: from synapse to perception. Physiol Rev 2006;86:1033-48.

[37] Caporale N, Dan Y. Spike timing-dependent plasticity: a Hebbian learning rule Annu Rev Neurosci 2008;31:25-46.

[38] Suppa A, Marsili L, Di Stasio F, Latorre A, Parvez AK, Colosimo C, et al. Primary motor cortex long-term plasticity in multiple system atrophy. Mov Disord 2014;29:97-104.

[39] Di Lazzaro V, Oliviero A, Pilato F, Saturno E, Dileone M, Mazzone P, et al. The physiological basis of transcranial motor cortex stimulation in conscious humans. Clin Neurophysiol 2004;115:255-66.

[40] Di Lazzaro V, Profice P, Ranieri F, Capone F, Dileone M, Oliviero A, et al. I-wave origin and modulation. Brain Stimul 2011;5:512-25.

[41] Dum RP, Strick PL. Motor areas in the frontal lobe of the primate. Physiol Behav 2002;77:677-82.

[42] Dum RP, Strick PL. Frontal lobe inputs to the digit representations of the motor areas on the lateral surface of the hemisphere. J Neurosci 2005;25: 1375-86.

[43] Dum RP, Levinthal DJ, Strick PL. The spinothalamic system targets motor and sensory areas in the cerebral cortex of monkeys. J Neurosci 2009;29: $14223-35$.

[44] Suppa A, Marsili L, Belvisi D, Conte A, Iezzi E, Modugno N, et al. Lack of LTPlike plasticity in primary motor cortex in Parkinson's disease. Exp Neurol 2011;227:296-301.

[45] Suppa A, Bologna M, Berardelli A. L-DOPA and cortical associative plasticity in Parkinson's disease. Clin Neurophysiol 2013b;124:638-9.

46] Suppa A, Huang YZ, Funke K, Ridding MC, Cheeran B, Di Lazzaro V, et al. Ten years of theta burst stimulation in humans: established knowledge, unknowns and prospects. Brain Stimul 2016;9:323-35.

[47] Suppa A, Bologna M, Conte A, Berardelli A, Fabbrini G. The effect of L-dopa in Parkinson's disease as revealed by neurophysiological studies of motor and sensory functions. Expert Rev Neurother 2017;17:181-92.

[48] Bologna M, Suppa A, Conte A, Latorre A, Rothwell JC, Berardelli A. Are studies of motor cortex plasticity relevant in human patients with Parkinson's disease? Clin Neurophysiol 2015;127:50-9.

[49] Jellinger KA. Pathology of Parkinson's disease. Changes other than the nigrostriatal pathway. Mol Chem Neuropathol 1991;14:153-97.

[50] Jellinger KA. Post mortem studies in Parkinson's disease - is it possible to detect brain areas for specific symptoms? J Neural Transm 1999;56:1-29. 
[51] Ueki Y, Mima T, Kotb MA, Sawada H, Saiki H, Ikeda A, et al. Altered plasticity of the human motor cortex in Parkinson's disease. Ann Neurol 2006;59: $60-71$.

[52] Bagnato S, Agostino R, Modugno N, Quartarone A, Berardelli A. Plasticity of the motor cortex in Parkinson's disease patients on and off therapy. Mov Disord 2006;21:639-45.

[53] Morgante F, Espay AJ, Gunraj C, Lang AE, Chen R. Motor cortex plasticity in Parkinson's disease and levodopa-induced dyskinesias. Brain 2006;129: 1059-69.

[54] Schwingenschuh P, Ruge D, Edwards MJ, Terranova C, Katschnig P, Carrillo F, et al. Distinguishing SWEDDs patients with asymmetric resting tremor from Parkinson's disease: a clinical and electrophysiological study. Mov Disord 2010;25:560-9.

[55] Kojovic M, Bologna M, Kassavetis P, Murase N, Palomar FJ, Berardelli A, et al. Functional reorganization of sensorimotor cortex in early Parkinson disease. Neurology 2012;78:1441-8.

[56] Kojovic M, Kassavetis P, Bologna M, Pareés I, Rubio-Agusti I, Berardelli A, et al. Transcranial magnetic stimulation follow-up study in early Parkinson's disease: a decline in compensation with disease progression? Mov Disord 2015;30:1098-106.

[57] Kačar A, Filipović SR, Kresojević N, Milanović SD, Ljubisavljević M, Kostić VS, et al. History of exposure to dopaminergic medication does not affect motor cortex plasticity and excitability in Parkinson's disease. Clin Neurophysiol 2013;124:697-707.

[58] Kawashima S, Ueki Y, Mima T, Fukuyama H, Ojika K, Matsukawa N. Differences in dopaminergic modulation to motor cortical plasticity between Parkinson's disease and multiple system atrophy. PLoS One 2013;8. e62515.

[59] Kishore A, Popa T, Balachandran A, Chandran S, Pradeep S, Backer F, et al. Cerebellar sensory processing alterations impact motor cortical plasticity in Parkinson's disease: clues from dyskinetic patients. Cereb Cortex 2014;24: 2055-67.

[60] Tsao H, Galea MP, Hodges PW. Reorganization of the motor cortex is associated with postural control deficits in recurrent low back pain. Brain 2008;131: $2161-71$.

[61] Tsao H, Galea MP, Hodges PW. Driving plasticity in the motor cortex in recurrent low back pain. Eur J Pain 2010;14:832-9.

[62] Schabrun SM, Christensen SW, Mrachacz-Kersting N, Graven-Nielsen T. Moto cortex reorganization and impaired function in the transition to sustained muscle pain. Cereb Cortex 2016;26:1878-90.

[63] Burns E, Chipchase LS, Schabrun SM. Altered function of intracortical networks in chronic lateral epicondylalgia. Eur J Pain 2016:20:1166-75.

[64] Apkarian AV, Bushnell MC, Treede RD, Zubieta JK. Human brain mechanisms of pain perception and regulation in health and disease. Eur J Pain 2005;9: $463-84$.

[65] Koyama T, McHaffie JG, Laurienti PJ, Coghill RC. The subjective experience of pain: where expectations become reality. Proc Natl Acad Sci U. S. A 2005;102: 12950-5.

[66] Iannetti GD, Niazy RK, Wise RG, Jezzard P, Brooks JC, Zambreanu L, et al. Simultaneous recording of laser-evoked brain potentials and continuous, high-field functional magnetic resonance imaging in humans. Neuroimage 2005:28:708-19.

[67] Tracey I, Mantyh PW. The cerebral signature for pain perception and its modulation. Neuron 2007;55:377-91.

[68] Tashev R, Belcheva S, Milenov K, Belcheva I. Antinociceptive effect of somatostatin microinjected into caudate putamen. Peptides 2001:22:1079-83.

[69] Braz JM, Nassar MA, Wood JN, Basbaum AI. Parallel "pain" pathways arise from subpopulations of primary afferent nociceptor. Neuron 2005;47: 787-93.
[70] Hagelberg N, Martikainen IK, Mansikka H, Hinkka S, Någren K, Hietala J, et al. Dopamine D2 receptor binding in the human brain is associated with the response to painful stimulation and pain modulatory capacity. Pain 2002;99: 273-9.

[71] Pertovaara A, Martikainen IK, Hagelberg N, Mansikka H, Någren K, Hietala J, et al. Striatal dopamine D2/D3 receptor availability correlates with individual response characteristics to pain. Eur J Neurosci 2004;20:1587-92.

[72] Martikainen IK, Hagelberg N, Mansikka H, Hietala J, Nagren K, Scheinin H, et al. Association of striatal dopamine D2/D3 receptor binding potential with pain but not tactile sensitivity or placebo analgesia. Neurosci Lett 2005;376: 149-53.

[73] Scott DJ, Heitzeg MM, Koeppe RA, Stohler CS, Zubieta JK. Variations in the human pain stress experience mediated by ventral and dorsal basal ganglia dopamine activity. J Neurosci 2006;26:10789-95.

[74] Scott DJ, Stohler CS, Koeppe RA, Zubieta JK. Time-course of change in [11C] carfentanil and $[11 \mathrm{C}]$ raclopride binding potential after a nonpharmacological challenge. Synapse 2007;61:707-14.

[75] Brefel-Courbon C, Payoux P, Thalamas C, Ory F, Quelven I, Chollet F, et al. Effect of levodopa on pain threshold in Parkinson's disease: a clinical and positron emission tomography study. Mov Disord 2005;20:1557-63.

[76] Magnusson JE, Fisher K. The involvement of dopamine in nociception: the role of $D(1)$ and $D(2)$ receptors in the dorsolateral striatum. Brain Res 2000;855: 260-6.

[77] Loher TJ, Burgunder JM, Weber S, Sommerhalder R, Krauss JK. Effect of chronic pallidal deep brain stimulation on off period dystonia and sensory symptoms in advanced Parkinson's disease. J Neurol Neurosurg Psychiatry 2002;73: 395-9.

[78] Dellapina E, Ory-Magne F, Regragui W, Thalamas C, Lazorthes Y, Rascol O, et al. Effect of subthalamic deep brain stimulation on pain in Parkinson's disease. Pain 2012;153:2267-73.

[79] Kim HJ, Jeon BS, Lee JY, Paek SH, Kim DG. The benefit of subthalamic deep brain stimulation for pain in Parkinson disease: a 2-year follow-up study. Neurosurgery 2012;70:18-23.

[80] Chudler EH. Response properties of neurons in the caudate-putamen and globus pallidus to noxious and non-noxious thermal stimulation in anesthetized rats. Brain Res 1998;812:283-8.

[81] Chudler EH, Dong WK. The role of the basal ganglia in nociception and pain. Pain 1995;60:3-38.

[82] Tinazzi M, Del Vesco C, Defazio G, Fincati E, Smania N, Moretto G, et al. Abnormal processing of the nociceptive input in Parkinson's disease: a study with CO2 laser evoked potentials. Pain 2008:136:117-24.

[83] Cruccu G, Aziz TZ, Garcia-Larrea L, Hansson P, Jensen TS, Lefaucheur JP, et al. EFNS guidelines on neurostimulation therapy for neuropathic pain. Eur J Neurol 2007;14:952-70.

[84] Lima MC, Fregni F. Motor cortex stimulation for chronic pain: systematic review and meta-analysis of the literature. Neurology 2008;70:2329-37.

[85] Nguyen JP, Nizard J, Keravel Y, Lefaucheur JP. Invasive brain stimulation for the treatment of neuropathic pain. Nat Rev Neurol 2011:7:699-709.

[86] Deer TR, Mekhail N, Petersen E, Krames E, Staats P, Pope J, et al. The appropriate use of neurostimulation: stimulation of the intracranial and extracranial space and head for chronic pain. Neuromodulation Appropriateness Consensus Committee. Neuromodulation 2014;17:551-70.

[87] Lefaucheur JP, André-Obadia N, Antal A, Ayache SS, Baeken C, Benninger DH, et al. Evidence-based guidelines on the therapeutic use of repetitive transcranial magnetic stimulation (rTMS). Clin Neurophysiol 2014:125:2150-206.

[88] Tomlinson CL, Stowe R, Patel S, Rick C, Gray R, Clarke CE. Systematic review of levodopa dose equivalency reporting in Parkinson's disease. Mov Disord $2010 ; 25: 2649-53$ 